\title{
A New Motion Planning Framework based on the Quantized LQR Method for Autonomous Robots
}

\author{
Onur Sencan \\ Department of Control and \\ Automation Engineering \\ Istanbul Technical University \\ Istanbul, Turkey 34398
}

\author{
Hakan Temeltas \\ Department of Control and \\ Automation Engineering \\ Istanbul Technical University \\ Istanbul, Turkey 34398
}

\begin{abstract}
This study addresses an argument on the disconnection between the computational side of the robot navigation problem with the control problem including concerns on stability. We aim to constitute a framework that includes a novel approach of using quantizers for occupancy grids and vehicle control systems concurrently. This representation allows stability concerned with the navigation structure through input and output quantizers in the framework. We have given the theoretical proofs of $q L Q R$ in the sense of Lyapunov stability alongside with the implementation details. The experimental results demonstrate the effectiveness of the qLQR controller and quantizers in the framework with realtime data and offline simulations.
\end{abstract}

Keywords-Robot motion; mobile robotics; hybrid systems; optimal control; quantization

\section{INTRODUCTION}

Systems that contain both continuous dynamics and discrete events are called hybrid or discontinuous dynamical systems. The discontinuity in the system can be based on the control system transitions like the operation of gearbox shift pattern in vehicles or the steep system dynamics like a change of direction or final stop of a bouncing ball. In the early studies of control theory, this well-known phenomenon in the system dynamics interpreted as a predictable disturbance [1] or the noise on the signal [2]. After the widespread usage of the pulse width modulation signals in electric drives at the beginning of the $90 \mathrm{~s}$, sliding mode control techniques $[3,4]$ use this nonlinear switching behavior as a control method for stabilizing nonlinear systems with an on-off(bang-bang) controller. Following the broad range of industrial practices, usage areas of sliding mode control reached many application areas in robotics like stabilization of autonomous surface vessels in rough open seas [5] and reactive position control of quadrotors [6]. Sliding mode control frameworks define the switching regions as discontinuity surfaces and aim to design feedback controllers that direct the optimal solutions of system states to settle around these surfaces. The near-optimal solutions around discontinuity surfaces induce chattering problem, which is high-frequency switching between various controllers that designed for different set points originated by neglected effects of actuator and system dynamics [7].

Projection of this chattering situation into robot navigation problem can be seen in a seesaw movement of a vehicle among attractive goal point and different repellent sources like the obstacles around the vehicle. At this point, derivative studies of artificial potential fields[8-10] and vector fields $[11,12]$ for navigation also tend to behave in the same manner around this instability boundaries. Different approaches like assigning coefficients for cost functions to stay away from these regions as in the dynamical window approaches $[13,14]$ do not guarantee a stabilizing controller and works only the predefined areas where the parameters are tuned for these special cases. Decentralized cooperative control of swarm robots as in [15] has related approaches but in a different context with a switched control system. This phenomenon of using existing control methods with different notation can be seen very often in the robotics literature. Similarly, optimal control theory literature dismisses antecedent study of Carathéodory's [16] and this leads up to related works on discontinuous dynamics with Carathéodory's solutions [17-19] and its variant Filippov solutions [20]. These studies suggest the equivalent solutions of Pontrayagin's minimum principle [21] by inspecting the vector fields of a specific operating point or neighborhood of an operating point respectively. Cortés [22] contributes a comprehensive review paper on discontinuous dynamics systems with Carathéodory's and Filippov's solutions. However, these approaches take a rather different way by focusing on the understanding of complex dynamics systems then discussing the stability or design control laws on discontinuous dynamics. The solutions of piecewise continuous vector fields are used in the area of state-dependent switching dynamical systems. In [23], the hybrid systems with sensor and actuator constraints are studied with a different notion called quantizers. It is evident that robotics applications are a perfect match for a hybrid system both with sensor and actuator constraints.

Quantization term is used to describe the levels or sets that are separated by discontinuous events like the change of gear in a vehicle. Quantized sets have their unique continuous dynamics, which may have similar properties like the shift change between second and third gears or completely different ones like switching between first and rear gears. Using quantization effect not as an error source to be predicted with white noise models, but rather as a stabilizer of an unstable discrete-time system by introducing the usage of quantized states as an input to state feedback mechanism is based on the study of Delchamps [24]. This strategy changes the traditional view of quantization from a simple rounding operator to a system state to be utilized in control system design process. The characteristics of quantization effects in hybrid systems are extensively studied in $[23,25]$. The proposed study is influenced by the idea of using the measurement quantization 
as an information coding of the real data, which is used directly by the controller. Also, in [26], quantization intervals are associated with the stability of the system in an explicit relation with the systems closed-loop unstable poles. This relation is exhibited by the minimum number of quantization levels and the system poles in the right-half plane (RHP). Therefore, it is essential to determine the minimum information that is needed for the higher levels of the control system after applying quantizer.

In this paper, we propose a navigation framework with input and output quantizers which are used to express sensor data in grid segmentations and generate reference inputs within motion planner to achieve asymptotic stability with quantized LQR(Linear Quadratic Regulator) respectively. A new LQR system namely qLQR is adapted from the quantizer definitions of [27] to a navigation framework with theoretical and simulation results. Using quantization on creating occupancy grids from sensor data and in control system architecture is a novel approach to robot navigation problem.

The paper organized as follows, first we give the quantization concept in control theoretical sense with some illustrations in Section II. In Section II-A, we introduce the general framework of input and output quantization for navigation. In Section II-B, the first quantizer in the framework, the input quantizer, is explained in detail how to handle path chunks from path planner with quantization. Following Section (II-C) is about the second quantizer in the framework, which is the output quantizer. In this section, we express how a quantizer is implemented into a control system with motion planner, which is responsible for generating reference control signals from the output of input quantizer block. In Section III, we derive the quantized LQR system for this framework, which is used in previous sections. In Section III-A, the quantized control structure is derived in classical control terms. In the next Section (III-B) we derive stability boundaries of control signals as the same structure in hybrid systems[23]. We show the effectiveness of $\mathrm{qLQR}$ compared to the traditional $\mathrm{LQR}$ system and qLQR usage in our framework both with real-time and simulation results.

\section{QuAntized FeEdBACK CONTROL}

The standard method for sampling a continuous error signal $e(t)$ to a sequence of impulse functions $e_{s}(t)$ achieved by a sampling switch with appropriately chosen sampling time $T$. The area of this impulse sequence is the sampling instant, which is denoted as $e(k T)$. Through the property of the area of Dirac-delta impulse function equals to 1 (i.e., $\int \delta(t) \mathrm{d} t=1$ ), the sampled error signal $e_{s}(t)$ is represented as,

$$
e_{s}(t)=\sum_{k=-\infty}^{\infty} e(k T) \delta(t-k T)
$$

To convert this sampling instants to a physical signal, there should be a holder which is engaged to hold the previous signal value until the next signal is sampled. Zero-order Holder is the simple and effective solution, denoted by $h_{0}$,

$$
h_{0}(k T)=e(k T)
$$

This sample and hold mechanism obeys the superposition rule. Thus, the linearity of the sampled continuous signal is not affected at this stage. In addition to that, a quantizer, which can also be interpreted as a nonlinear discontinuous sampler is added just before the system. The quantization operation is a mapping operator, that is directed from a Euclidean space $\Re^{k}$ to a finite subset of this space $\mathcal{P}_{\text {fin }}\left(\Re^{k}\right)$,

$$
q: \Re^{k} \mapsto \mathcal{P}_{\text {fin }}\left(\Re^{k}\right)
$$

The operation introduced with a quantization operator $q$ sets the sampled values to corresponding quantized set values. In Fig. 1 sampling, hold and quantization operations are illustrated together. Here, the quantizer operation for this example defined as,

$$
q(x):=\left\{\begin{array}{lll}
L 1 & 0 \leqslant h_{0}(k T) \leqslant l 1 & (\operatorname{set} A) \\
L 2 & l 1 \leqslant h_{0}(k T) \leqslant l 2 & (\operatorname{set} B) \\
L 3 & l 2 \leqslant h_{0}(k T) \leqslant l 3 & (\operatorname{set} C)
\end{array}\right.
$$

Quantizer operator is arranged to switch between three different sets $A, B$ and $C$ depending on the value of signal against intervals $l 1, l 2, l 3$ and get corresponding set values, which are $L 1, L 2, L 3$.

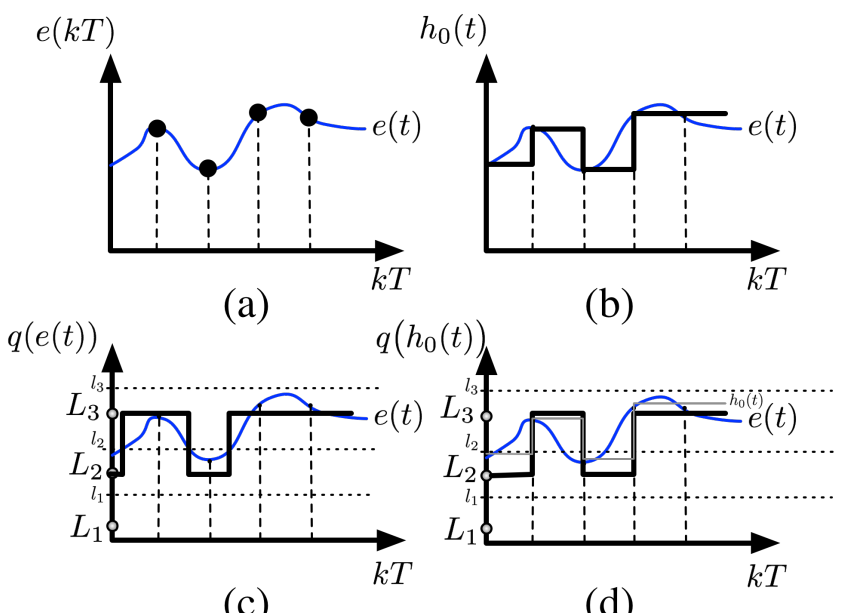

(c)

(d)

Fig. 1. Sampling, hold and quantization operations on a random error signal. (a) Discrete sampler (b) Zero order hold operator (c) Quantization operator applied on continuous signal (d) Quantizer operator applied on hold signal.

The unified framework considers quantization, time delay and disturbance to handle nonlinearities in system dynamics even the system is modeled or behaved like a linear system. Thus, the first step is modeling the additive nonlinear effects in deterministic error signals $(e)$, such as Gaussian white noise. The second step is to design a control law $k$ disregarding the error signals with static feedback. Generalized dynamic equations for such a robotics system can be written as;

$$
\begin{aligned}
\dot{x} & =f(x, u) \\
y & =h(x)
\end{aligned}
$$

where, $x \in \Re^{n}$ for system states, $u \in \Re^{m}$ is control signal, $y \in \Re^{l}$ is the system output, $f$ is the nonlinear system characteristic function. $h$ is the nonlinear measurement 
function. We aim to design a control law $k$ that asymptotically stabilizes the given system, $u=k(x)$.

Now suppose that the state feedback $x$ is quantized, hence the control law becomes

$$
\begin{array}{r}
u=k(q(x)) \\
u=k(x+e)
\end{array}
$$

here $e:=q(x)-x$ is quantization error. The goal is to reduce the $e$ with time goes to infinity;

$$
\mathbf{G}: \lim _{t \rightarrow \infty} e(t)=0
$$

\section{A. General Navigation Framework with Quantized Feedback Control System}

The principal objective of using quantizers is the robustness of the controllers under measurement and modeling errors. This purpose is achieved via input to state stability(ISS), which uses Lyapunov functions and small-gain theorem. The quantized variable can be any signal like measurement output, control input or state variable. The quantizer maps these continuous variables to their quantized conjugates. Next sections explain that how we implement this quantization operator to describe grid maps in input quantizer and generate reference values and control a stabilizable control system with discontinuous dynamics of steering and throttle subsystems through output quantizer. The generalized framework is shown in Fig. 2.

Obstacle maps layer is used for pre-occupation of the 3D sensor data into initial grid layout in raw form or after some outlier elimination process $[28,29]$. Traffic planner block stands for the infrastructural decision logic and constraints like a temporary stop in traffic lights and in the intersection points or speed limits in different roads which are also resources of discontinuity in navigation problem. Input quantizer samples the input sensors as an input signal to path and motion planner by taking the static and dynamic obstacles and the current vehicle states into consideration. The output of the motion planner gives a maneuverable path, which is formed from grid cells. Each grid cell is a shaped reference input to the system. At this point, the second quantizer becomes a part of the system, which is called as output quantizer. Output quantizer converts the motion planner outputs like linear and angular velocities to the discrete levels available. Output quantizer provides reference inputs to the low-level controllers in the last layer. These controllers are typical PID controllers with protections like anti-windup, saturation delimiters.

\section{B. Input Quantizer}

There are two stages here that need to be considered for stabilization of the system under quantization. One of them is the quantization levels, which are dynamic in the creation of occupancy grids in Region of Interest(ROI) and static in vehicle control systems. The second stage is to find a feedback control law that stabilizes the system. First, we derive the system model, which is the mathematical model of Ackermann type autonomous guided vehicle(AGV). The vehicle kinematics in Fig. 3 is given as a nonlinear function $f$, subject to state and control variables(or constraints)

$$
\dot{x}=f(x, u)
$$

The vehicle state variables are $x, y$, the locations in $\mathrm{x}-\mathrm{y}$ coordinates, $\theta$ orientation with linear velocity $\nu$ and steering angle $\varphi$. Control variables of the vehicle are linear acceleration $a$ and angular velocity $\omega$. In vector notation:

$$
\mathbf{x}=\left[\begin{array}{c}
x \\
y \\
\theta \\
\nu \\
\varphi
\end{array}\right], \quad \mathbf{u}=\left[\begin{array}{l}
a \\
\omega
\end{array}\right]
$$

Eventually, the open form of the nonlinear system dynamics given in (9) can be derived as follows:

$$
\dot{\mathbf{x}}=\frac{\mathrm{d}}{\mathrm{d} t}\left[\begin{array}{c}
x \\
y \\
\theta \\
\nu \\
\varphi
\end{array}\right]=\left[\begin{array}{c}
\nu \cos (\theta) \\
\nu \sin (\theta) \\
\nu \tan (\varphi) \\
a \\
\omega
\end{array}\right]
$$

Quantization process on vehicle state variables expressed as follows:

$$
\begin{gathered}
x \leftarrow G_{x}\left(x_{c}\right), y \leftarrow G_{y}\left(y_{c}\right), z \leftarrow G_{z}\left(z_{c}\right) \\
\psi \leftarrow \arctan 2\left(m_{y}, m_{x}\right), \phi \leftarrow \arctan 2\left(m_{z}, m_{x}\right), \\
\theta \leftarrow \arctan 2\left(m_{z}, m_{y}\right)
\end{gathered}
$$

In this assignment, state variables that hold the position for every axes, get values from the current vehicle position in corresponding Graph tree that is shaped from path and motion planners. Roll, pitch, yaw angles of the vehicle get values from the tangents of the path spline. This assignment visualized in Fig. 4.

Input planner may also return feedback to the path planner if the minimum gridding length is exceeded. The mission of the input quantizer is to divide the region into grid partitions, which are independent for each axis. The detailed input quantizer block of the framework is given in Fig. 5.

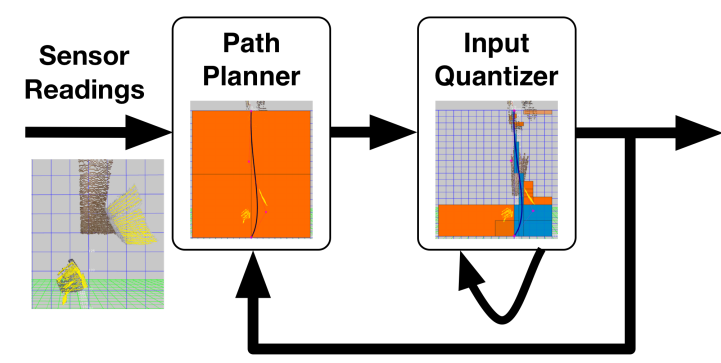

Fig. 5. Input quantizer and path planning blocks.

Division starts with coarse parent grids. Grids are assigned to the path (blue tetragons) if segmented path chunks (generated by using one of RRT planners in [30-33]) are located in grid regions. Then, the obstacles are initiated to the system with external sensors (in our case, a Kinect 3D sensor). Some of the grids are occupied (red tetragons) if there is an obstacle in grid region. In each step, the grids are become denser with splitting a coarse grid into a new grid with the half size (not necessary to be symmetrical in all axes). This division is 


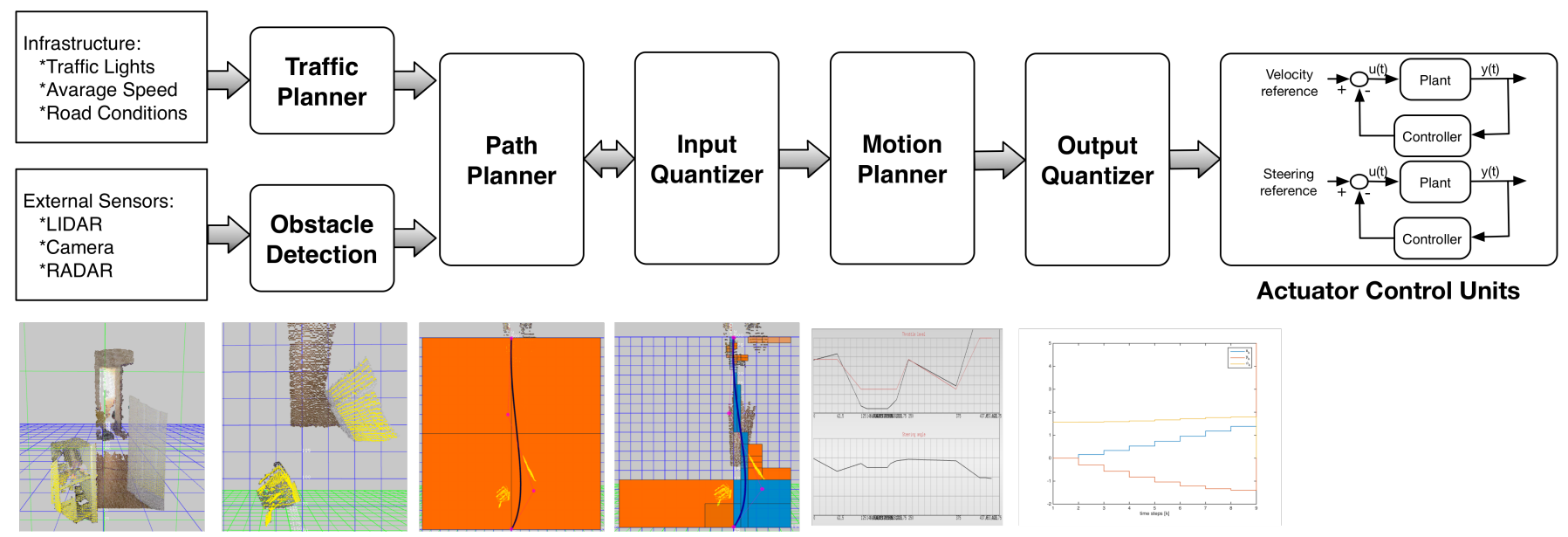

Fig. 2. General framework of the quantized feedback control system.

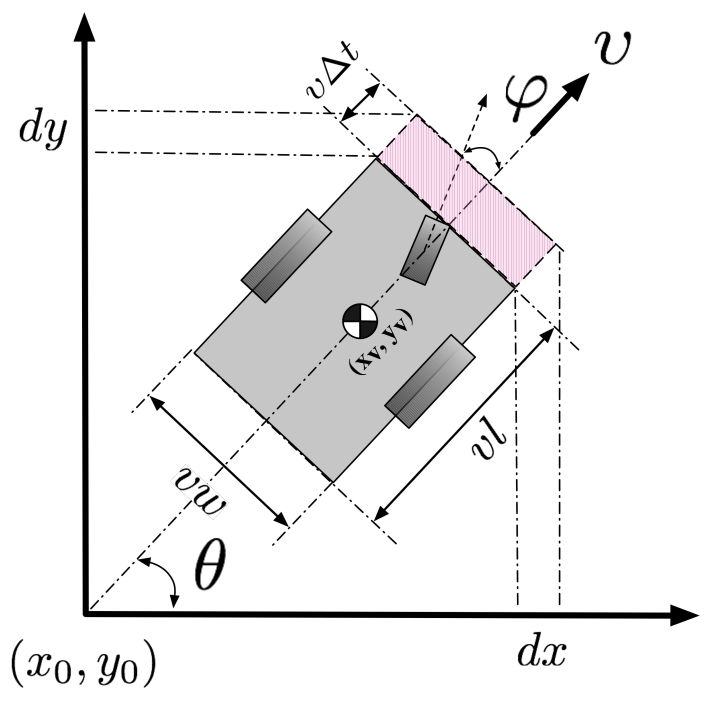

Fig. 3. Ackermann type steering model of AGV.

repeated till the occupied obstacle grids, and grids associated to the path chunks are not are not overlapped (Fig. 6). All grids are symbolized with a graph tree data structure, which is independent in each axis. If the overlapping still exists to the minimum grid size allowed (which is determined by the steering and throttle actuator constraints, vehicle dimensions, etc.), then a feedback signal is generated to a new route among the alternative solutions.

The first path is initiated through the internal processes of the selected path planner algorithm. However, the afterward process is executed with the cooperation of the input quantizer. Grid segmentation logic is shown in Fig. 7.

\section{Output Quantizer}

The output quantizer of the controller takes reshaped quantized reference inputs from motion planner and generates

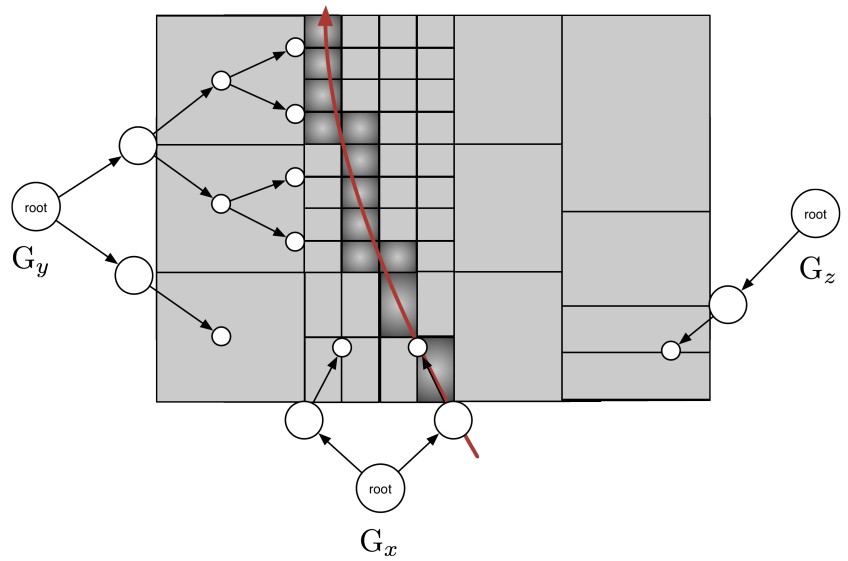

Fig. 4. Assignment of the sensor inputs in input quantization block.

outputs with regard to controllers sampling levels.After finding a non-overlapping grid segmentations through the selected path, then the grids are used in the motion planner section to generate velocity and steering angle references. Intuitively, if the path goes through in a close neighborhood of an obstacle, then the grids are denser in those areas. Also, the steering angle has a value between the tangent of grids and the tangent of the spline. Those references are selected from a suitable set of the actuator capabilities. After the motion planner step, we obtain the reference values to be tracked. However, we should also maintain the stability of the system while sweeping through the reference values. At this stage, the second quantizer type in the system, the output quantizer becomes a part of the framework. Connection between these blocks are illustrated in Fig. 8. 


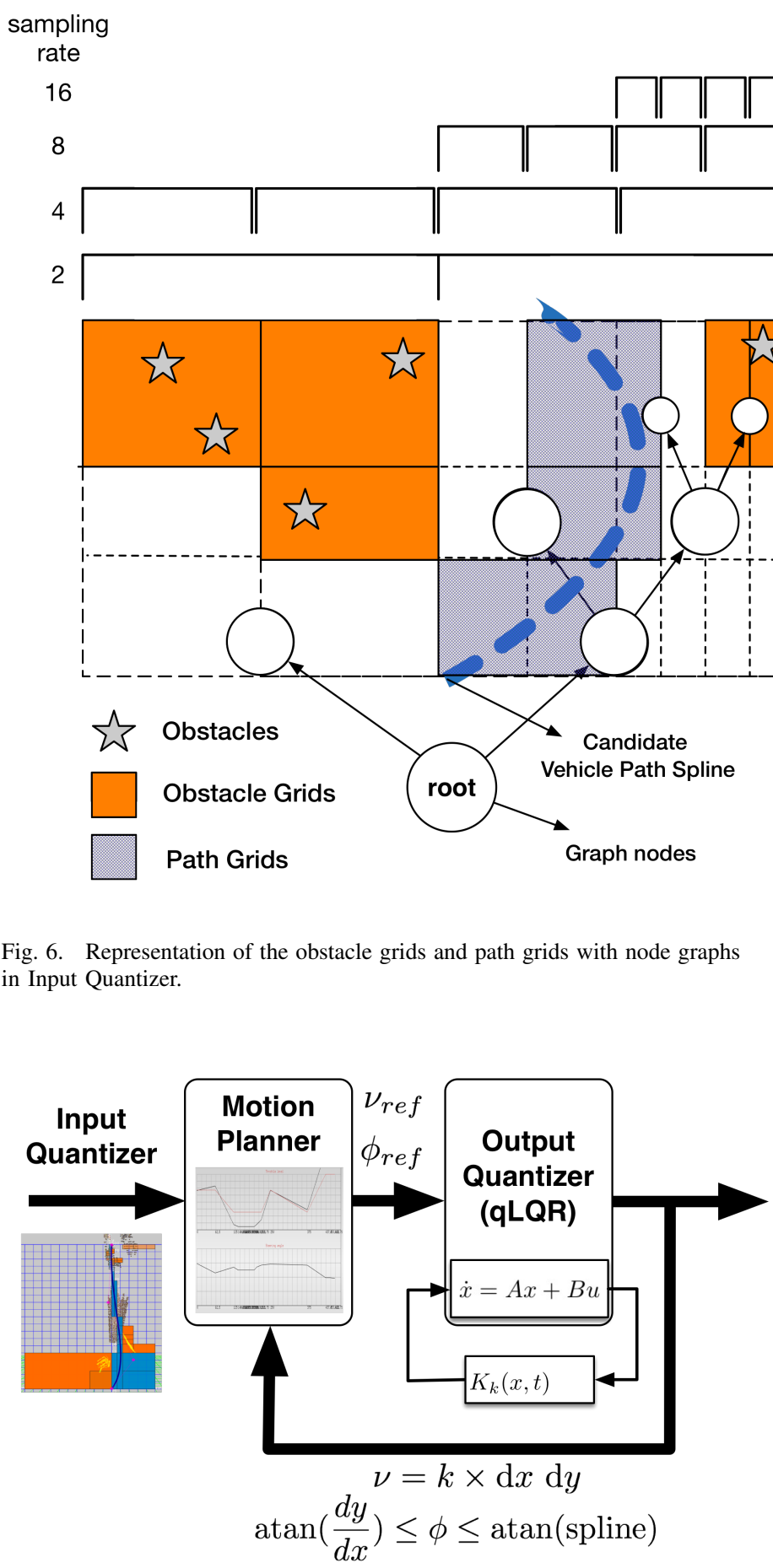

Fig. 8. Motion planner and output quantizer blocks.

In the simplest case, with an Ackerman type vehicle, there are two control variables $\nu, \omega$ linear and angular velocities respectively. If we need a smooth ride, then we need to use the derivatives $\dot{\nu}, \dot{\omega}$. Linear velocity of the vehicle can be assigned as a fixed value like the many approaches in the literature. However, in this approach grid size of each axis promises a throughput that can be used to select the optimal velocity. To do this, we first define an expected mean value of the velocity

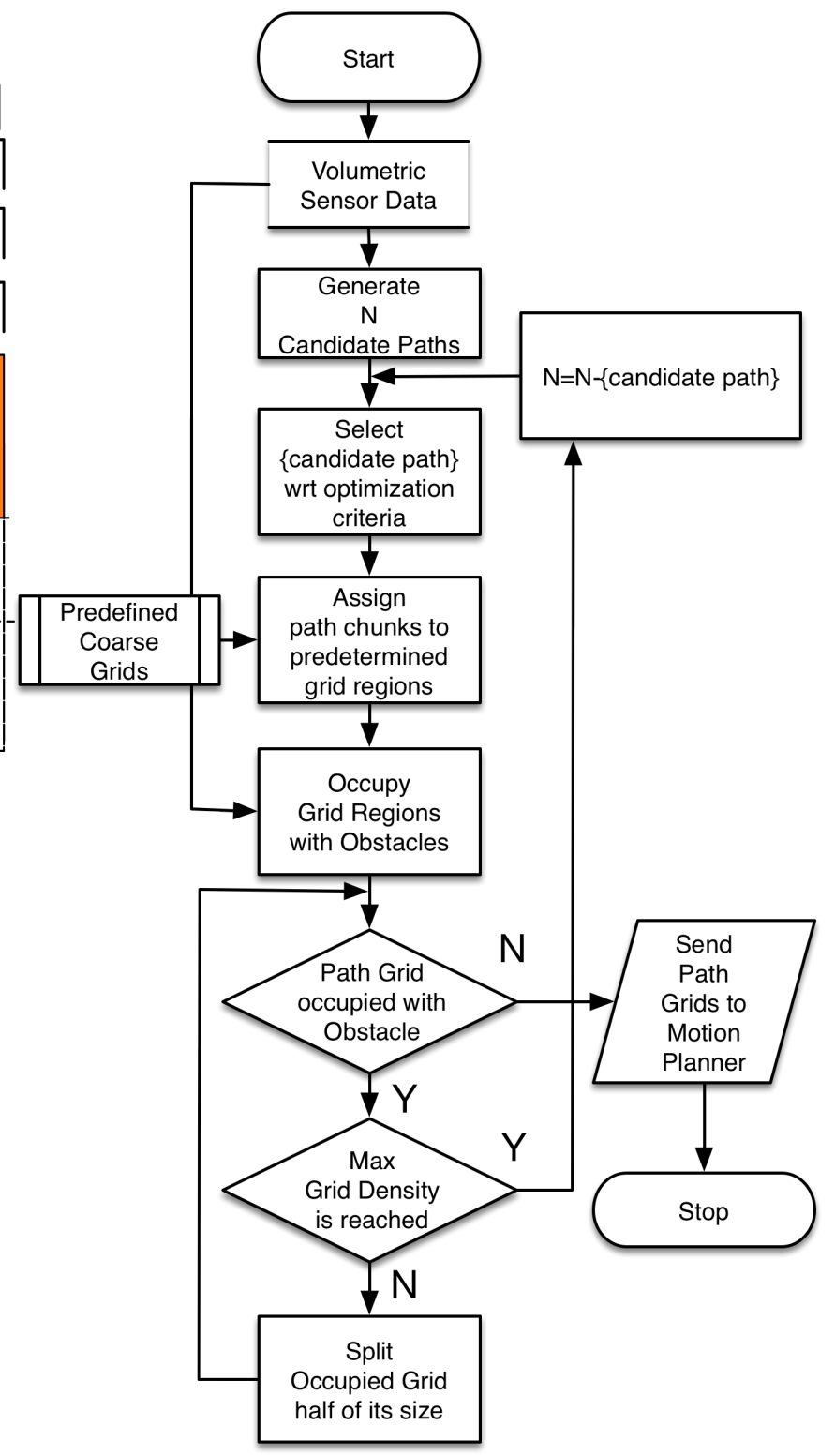

Fig. 7. Flow chart of the grid segmentation logic.

$\bar{\nu}$ in terms of grid structure,

$$
\bar{\nu}=\frac{\sqrt{\left(d y_{0}+d y^{+}\right)^{2}+\left(d x_{0}+d x^{+}\right)^{2}}}{\Delta \mathrm{t}}
$$

Here, $d x_{0}, d y_{0}$ are the spatial samplers of the current grid and $d x^{+}, d y^{+}$are the spatial samplers of the next grid that is predicted in motion controller (Fig. 9).

Quantized value of the expected mean velocity is denoted as $\lfloor\bar{\nu} \mid$. This special floor operator is a direct consequence of the vehicle throttle levels which is shown with an example with a gear-shift mechanism in Fig. 10. 


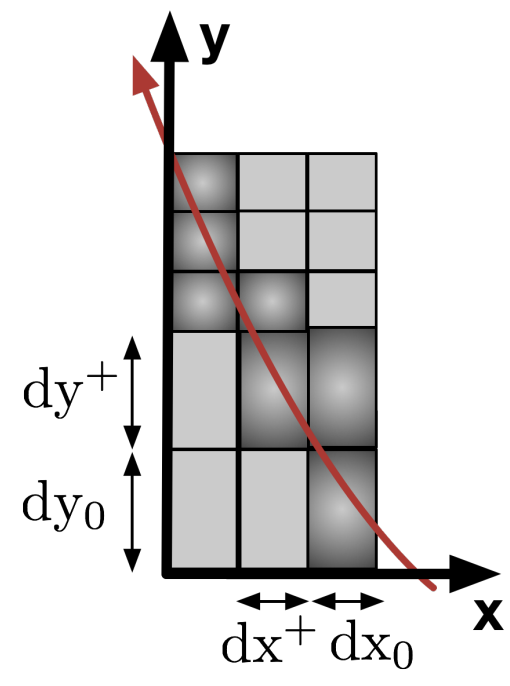

Fig. 9. Visualization of the parameters in expected mean velocity calculation

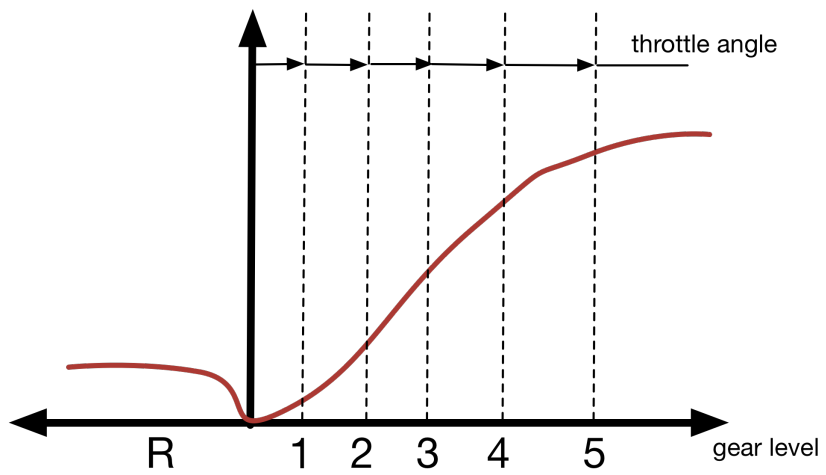

Fig. 10. Throttle with a gear-shifting is an example of switched system.

Heading angle of the vehicle is either can be found by using the tangent of the curvature (i.e., $\psi=\left\lfloor\arctan 2\left(m_{y}, m_{x}\right)\right\rfloor$ ) or tangent of sequential grids (i.e., $\psi=\left\lfloor\arctan 2\left(\frac{d y_{0}+d y^{+}}{d x_{0}+d x^{+}}\right)\right\rfloor$). The latter one is definitely a coarse estimation than the former one. State space of the closed-loop system with these control inputs can be found as follows:

$$
\begin{aligned}
x_{k+1} & =A x_{k}+B q_{k} \\
Q\left(y_{k}\right) & =C x_{k}
\end{aligned}
$$

Here, $q_{k}=Q\left(u_{k}\right)$ is quantized output of the output quantizer to the controller. Output quantizer signal with state feedback is found as,

$$
\begin{aligned}
& u_{k}=r_{k}+K x_{k} \\
& r_{k}=\left[\begin{array}{l}
\lfloor\bar{\nu}\rfloor_{V_{t}} \\
\lfloor\bar{\psi}\rfloor_{V_{s}}
\end{array}\right]
\end{aligned}
$$

$\mathrm{K}$ is gain matrix that gives us the flexibility in controller design. Special floor functions \lfloor\rfloor$_{V_{t}}$ and \lfloor\rfloor$_{V_{s}}$ quantize the reference output from motion planner to the system specific set levels. Here the optimization criteria is minimizing the quantized values of the system output(i.e., state variables where $\mathbf{C}=\mathbf{I}(n)$ ) which is,

$$
\begin{array}{ll}
\underset{e r r o r}{\operatorname{minimize}} & y_{k}-Q\left(y_{k}\right) \\
\text { subject to } & \dot{x}=f(x, u) .
\end{array}
$$

Starting from this point of view, state space equations can be written as follows:

$$
\begin{aligned}
x_{k+1} & =\mathbf{A} x_{k}+\mathbf{B} q_{k} \\
& =\mathbf{A} x_{k}+\mathbf{B}\left(r_{k}+\mathbf{K} x_{k}\right) \\
& =(\mathbf{A}+\mathbf{B K}) x_{k}+\mathbf{B} r_{k} \\
& =\mathbf{A}_{c l} x_{k}+\mathbf{B} r_{k}
\end{aligned}
$$

Here $\mathbf{A}$ is a constant valued system matrix and relates the state changes with previous states. The term $\mathbf{B}$ is the control matrix and has dependency on heading angle $\psi$. Apparently, with static quantization levels of the controller denoted by $r_{k}$ the only alternative that we can control is $\mathbf{K}$ gain matrix. By changing values of the gain matrix, we can determine the eigenvalues of the closed-loop system. The system which is shown with linear state space model is asymptotically stable if all eigenvalues of the $\mathbf{A}$ is negative. This condition is equivalent to the following Lyapunov equation in terms of Lyapunov stability.

$$
\mathbf{A}^{\mathrm{T}} \mathbf{P}+\mathbf{P A}=-\mathbf{Q}
$$

Here $\mathbf{P}$ is positive definite Hermitian matrix and $\mathbf{Q}$ is positive definite to make left hand side negative definite equation. The corresponding Lyapunov function is,

$$
V(x)=x^{\mathrm{T}} \mathbf{P} x
$$

Consider a linear system,

$$
\dot{x}=\mathbf{A} x+\mathbf{B} u
$$

where $x \in \mathbb{R}^{n}, u \in \mathbb{R}^{m}$. Suppose this system is stabilizable with a gain matrix $\mathbf{K}$ and with a feedback control law $u=K x$. State space model of the closed-loop system is now,

$$
\begin{aligned}
\dot{x} & =\mathbf{A} x+\mathbf{B} u \\
& =\mathbf{A} x+\mathbf{B K} x \\
& =(\mathbf{A}+\mathbf{B K}) x \\
& :=\mathbf{A}_{c} x
\end{aligned}
$$

Applying the Lyapunov stability equation in (18) to closedloop stabilizable control system under the conditions $B \neq 0$, $K \neq 0$ gives,

$$
\begin{aligned}
\mathbf{A}_{c}^{\mathrm{T}} \mathbf{P}+\mathbf{P A} \mathbf{A}_{c} & =-\mathbf{Q} \\
(\mathbf{A}+\mathbf{B K})^{\mathrm{T}} \mathbf{P}+\mathbf{P A}+\mathbf{B K} & =-\mathbf{Q}
\end{aligned}
$$

Parameters $\lambda_{\min }, \lambda_{\max }$ are the minimum and maximum eigenvalues of the system respectively. These are decisive parameters of the system boundaries and hold for following inequality.

$$
\lambda_{\min }(P)|x|^{2} \leq x^{\mathrm{T}} P x \leq \lambda_{\max }(P)|x|^{2}
$$


The feedback law cannot be implemented directly because of the quantization takes place in our system. Quantizer $q_{\mu}($. is defined for a variable $z$ as,

$$
q_{\mu}(z):=\mu q_{\mu}\left(\frac{z}{\mu}\right)
$$

$\mu$ here is a strictly positive scalar value $(\mu>0)$. We can think $\mu$ as a zoom variable as in [23]. Increasing $\mu$ yields coarser quantization, which increases the ROI and at the same quantization error. Conversely, decreasing $\mu$ leads to denser quantization that decreases the quantization error but limits the range. The range of quantizer defined as $R O I=R \mu$ and quantization error with $\Delta \mu$. Proof of asymptotic stability holds for zoom-in/out cases given in Appendix.

The output quantizer can be formed as a state feedback,

$$
u=K q_{\mu}(x)
$$

For a closed-loop system, derivation of the quantized version of (21) is as follows:

$$
\begin{aligned}
\dot{x} & =\mathbf{A} x+\mathbf{B} u \\
& =\mathbf{A} x+\mathbf{B K} \mu q_{\mu}\left(\frac{x}{\mu}\right) \\
& =(\mathbf{A}+\mathbf{B K}) x+\mathbf{B K} \mu\left(q_{\mu}\left(\frac{x}{\mu}\right)-\frac{x}{\mu}\right)
\end{aligned}
$$

To inspect the behaviour of the system stability, we should check the trajectories of the system given in (26). Principally, we should define the overbounding polytope of stability regions of the state space. This ball $B_{1}$ is associated with the state ranges which is defined with $R \mu$ before.

$$
B_{1}:=\{x:|x| \leq R \mu\}
$$

Second polytope is defined to specify the lower boundary of the state space. To define this, first one should use the Lyapunov stability theorem. Suppose there is a Lyapunov function $V$, which has the following gradient,

$$
\dot{V}(x) \leq 0, \forall x \neq 0, \dot{V}(0)=0
$$

The general interpretation of the Lyapunov function $V$ as a generalized energy function which is always loses energy(except at origin). For this system, Lyapunov function defined as in (19).

$$
V(x)=x^{\top} \mathbf{P} x
$$

Substituting closed-loop system in (26) in (28) satisfies,

$$
\begin{aligned}
\dot{V}(x) & =-x^{\top} \mathbf{Q} x+2 x^{\top} \mathbf{P B K} \mu\left(q_{\mu}\left(\frac{x}{\mu}\right)-\frac{x}{\mu}\right) \\
& \leq-\lambda_{\min }(Q)|x|^{2}+2|x|\|\mathbf{P B K}\| \Delta \\
& \leq-|x| \lambda_{\min }(Q)\left(|x|-\Theta_{x} \Delta\right)
\end{aligned}
$$

where,

$$
\Theta_{x}:=\frac{2\|\mathbf{P B K}\|}{\lambda_{\min }(Q)}
$$

For a small $\epsilon>0$, we can set boundaries for the system states,

$$
\Theta_{x} \Delta(1+\epsilon) \leq|x| \leq R \mu
$$

Therefore, rate of change of the Lyapunov function in (30) has a lower limit which is bounded by the lower thresholds of the state variables. This lower limit constitutes the second polytope $B_{2}$,

$$
B_{2}:=\left\{x:|x| \leq \Theta_{x} \Delta(1+\epsilon)\right\}
$$

Ellipsoid for the operating point is constructed as,

$$
R_{1}(\mu):=\left\{x: x^{\top} \mathbf{P} x \leq \frac{\lambda_{\min }(\mathbf{P}) M^{2} \mu^{2}}{\|\mathbf{K}\|^{2}}\right\}
$$

Ellipsoids for attraction regions are defined as,

$$
R_{2}(\mu):=\left\{x: x^{\top} \mathbf{P} x \leq \lambda_{\max }(\mathbf{P}) \mathbf{Q}^{2} \Delta^{2}(1+\epsilon)^{2} \mu^{2}\right\}
$$

These regions are illustrated in Fig. 11

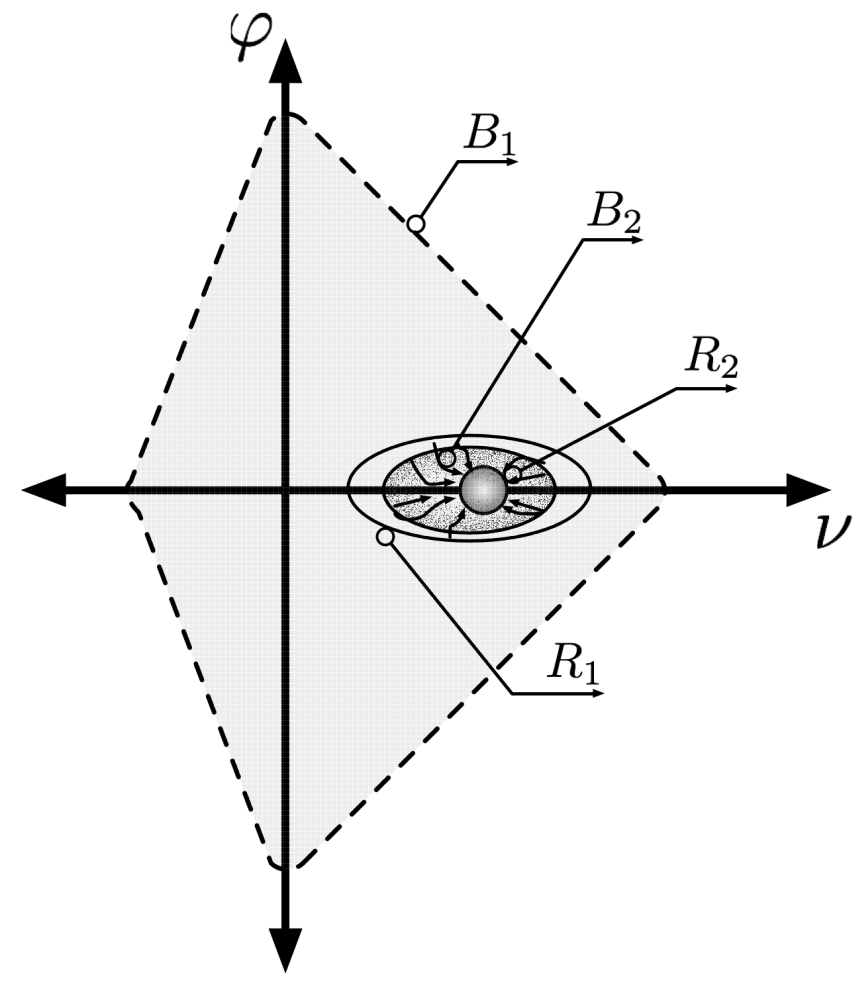

Fig. 11. Boundary polytopes of control variables for Lyapunov stability $B_{1}$ region denotes the state minimum and maximum values(max velocity and steering angles). $R_{1}$ is the selected operating or linearization point of vehicle dynamics. $R_{2}$ is the attraction(both appear as discontinuity or stability surface) region. $B_{2}$ shows the rate of change constraints of the state variables, because speed and steering angle are dependent variables in terms of stability analysis.

All solutions initialized in region $R_{1}(\mu)$ will be entered to inner region $R_{2}(\mu)$ in finite dwell time given by the following formula in [23]

$$
T:=\frac{\lambda_{\min }(\mathbf{P}) M^{2}-\lambda_{\max }(\mathbf{P}) \mathbf{Q}^{2}\|\mathbf{K}\|^{2} \Delta^{2}(1+\epsilon)^{2}}{\mathbf{Q}^{2}\|\mathbf{K}\| \Delta^{2} \epsilon(1+\epsilon) \lambda_{\min }(\mathbf{Q})}
$$


Asymptotic stability proof for a quantized feedback controller is given in [23]. Adaptation of this proofs to our framework is given in Appendix section.

Output quantizer layer is the most sophisticated part of the framework in control theoretical way. In Section III, an adaptation of hybrid control systems in [27] quantized LQR controller is indroduced as a stabilizable controller for the framework, when there is traditional LQR controller can be found for the system (for a controllable and stabilizable system). The reason behind that we use a quantized LQR controller instead of a traditional LQR controller is to take account of the reference levels that can be able to follow by the low-level actuators. Without considering the low-level capability of the system, one is always to be mistaken on the assumption of a perfect lowlevel actuation system, and this causes undesirable results in real life scenarios. One of the most faced situation is switching systems, where from its nature, switching between two stable modes of control operations may cause an unstable behavior. Conversely, switching between two unstable modes may cause a stable operation contrary to the instincts.

\section{QUANTIZED LQR FOR OUTPUT QUANTIZER}

In this section, we derive the control system which is denoted as $\mathrm{K}$ in previous sections for output quantizer in the framework. First, we explain the idea of stabilization for traditional Linear Quadratic Regulator(LQR) in discrete systems and expand it to the new approach of quantized LQR(q-LQR) structure. Both theoretical results and simulation results are given in order.

\section{A. Optimal Control for Discrete System}

Discrete time system with $\mathrm{k}$ sampling steps is defined as follows,

$$
x_{k+1}=\mathbf{A} x_{k}+\mathbf{B} u_{k}
$$

with given initial condition $x_{0}$. The design objective is to find an optimal control $u_{k}^{*}$ so that the performance index $J$, which is designed for the control demands,

$$
J=\frac{1}{2} x_{N}^{T} S_{N} x_{N}+\frac{1}{2} \sum_{k}^{N-1} x_{k}^{T} Q x_{k}+u_{k}^{T} R u_{k}
$$

can be minimized. Here $N$ is the final time, $S, Q$ and $R$ are the weight coefficient matrices for the final state, runtime states and the control inputs. To solve this optimization problem, first we need to derive a Hamiltonian function $H_{k}$ with Lagrange multiplier $p$,

$H_{k}\left(x_{k}, p_{k+1}, u_{k}\right)=\frac{1}{2} x_{k}^{T} Q x_{k}+\frac{1}{2} u_{k}^{T} R u_{k}+p_{k+1}^{T} A x_{k}+p_{k+1}^{T} B u_{k}$

The necessary conditions where the performance index is to be a minima or maxima (i.e. optimum) are given as follows:

$$
\begin{aligned}
& \frac{\partial H_{k}}{\partial u_{k}^{*}}=0=R u_{k}^{*}+B^{T} p_{k+1}^{*} \\
& \frac{\partial H_{k}}{\partial p_{k}^{*}}=x_{k+1}^{*}=A x_{k}^{*}+B u_{k}^{*} \\
& \frac{\partial H_{k}}{\partial x_{k}^{*}}=p_{k}^{*}=Q x_{k}^{*}+A^{T} p_{k+1}^{*}
\end{aligned}
$$

Optimal control function for given discrete system can be found from (39) as,

$$
u_{k}^{*}=-R^{-1} B^{T} p_{k+1}^{T}
$$

Substituting the optimal control signal in (42) to (40) gives,

$$
x_{k+1}^{*}=A x_{k}^{*}-B R^{-1} B^{T} p_{k+1}^{*}
$$

Using (41) and (43), we can show the feature states and the Lagrange multipliers in left hand side,

$$
\begin{aligned}
x_{k+1}^{*} & =A x_{k}^{*}-B R^{-1} B^{T} p_{k+1}^{*} \\
A^{T} p_{k+1}^{*} & =p_{k}^{*}-Q x_{k}^{*}
\end{aligned}
$$

Conjugated equations (44) and (45) are difficult to solve. However, there is a special solution for this case,

$$
p_{k}=S_{k} x_{k}
$$

Rearranging (44) with (46) gives,

$$
x_{k+1}^{*}=\left(I-B R^{-1} B^{T} S_{k+1}\right)^{-1} A x_{k}^{*}
$$

It can be shown that the $S_{k+1}$ is positive semi-definite and thus inverse will always exist. Substituting the $p_{k}$ term in (46) into (45) brings us,

$$
A^{T} S_{k+1} x_{k+1}^{*}=\left(S_{k}-Q\right) x_{k}^{*}
$$

By using (48) with (47),

$$
A^{T} S_{k+1}\left(I-B R^{-1} B^{T} S_{k+1}\right)^{-1} A x_{k}^{*}=\left(S_{k}-Q\right) x_{k}^{*}
$$

If one inspect (49), that can bee seen the equation is independent from the states i.e.,

$$
A^{T} S_{k+1}\left(I-B R^{-1} B^{T} S_{k+1}\right)^{-1} A=\left(S_{k}-Q\right)
$$

Equation (50) is called as Discrete Riccati Equation. Here, $S_{k}$ is referred as Riccati. To find the optimal control, substituting (46) to (42) gives us,

$$
u_{k}^{*}=-R^{-1} B^{T} S_{k+1} x_{k+1}^{*}
$$

with substitution $x_{k+1}$ term of (47) into (51),

$$
\begin{aligned}
u_{k}^{*} & =-R^{-1} B^{T} S_{k+1}\left(I-B R^{-1} B^{T} S_{k+1}\right)^{-1} A x_{k}^{*} \\
& =-\left(R+B^{T} S_{k+1}\right)^{-1} B^{T} S_{k+1} A x_{k}^{*} \\
& =-K_{k} x_{k}^{*}
\end{aligned}
$$

here $K_{k}$ is the state feedback coefficient matrix for the optimal controller,

$$
K_{k}=\left(R+B^{T} S_{k+1} B\right)^{-1} B^{T} S_{k+1} A
$$

Also, the Riccati term can be extracted using (53) and (50),

$$
S_{k}=Q+A^{T} S_{k+1}\left(A-B K_{k}\right)
$$

Some rule of the thumb information for performance index are 
- Increasing the $Q$, increases the bandwidth.

- Increasing only the diagonals of the $Q$, increases the damping ratio.

- Response of the state $x_{j}$ can be made faster by increasing the diagonal entry $q_{j j}$ in the weight matrix $Q$.

\section{B. Quantized Linear Quadratic Regulator as Minimum Energy Controller}

If a LTI system $[A, B]$ is stabilizable, it is also quadrically stabilizable. Thus, there is a state-feedback control input $u$ that shapes the states, performs a decreasing Lyapunov function, which is an indicator of the stabilization. These Lyapunov functions are called control Lyapunov functions (CLF). A quadratic CLF is constituted by,

$$
V(x)=x_{k}^{T} S_{k} x_{k}
$$

The gradient of Lyapunov function is denoted as $\Delta V(x)$ and it should always have a negative sign if $V(x)$ is a continuously decreasing function.

$$
\Delta V(x):=V\left(x_{k+1}^{*}\right)-V\left(x_{k}^{*}\right)<0
$$

Now, we convert our problem to find a control set $U$ that minimizes the given CLF in (55),

$$
U:\left\{u_{i} \in \mathbb{R} ; i \in \mathbb{Z}\right\}
$$

a quantizer is a function that maps the state set $X$ to the control set $U$ with one to one correspondence,

$$
f: X \mapsto U
$$

where,

$$
X:\left\{x_{i} \in \mathbb{R} \mid f(x)=u_{i}, i \in \mathbb{Z}\right\}
$$

Substituting the quantizer into (56) and using the discrete system equation $x_{k+1}=A x_{k}+B u_{k}$ we have,

$$
\Delta V(x)=V\left(A x_{k}+B f\left(x_{k}\right)\right)-V\left(x_{k}\right)<0
$$

The problem of the quantization is to find the minimum coarsest control level that stabilizes the system. So, let $\rho$ a multiplier on the unit control set $U$. Aim is to find the $\rho$ value that minimizes the performance index,

$f: X \mapsto \beta U, X:\left\{x_{i} \in \mathbb{R} \mid f(x)=\beta u_{i} ; i \in \mathbb{Z}, \beta \in \mathbb{R}, \beta>0\right\}$

Equation (57) shows there is no loss of generality with the scaling[26] and hereafter, the $\beta$ value is assumed to be $\beta=1$ and CLF is a robust CLF for these given fixed control values. Like the expanded version of normal LQR controllers in (51), same equation can be written for the quantized LQR controller;

$$
u_{k}^{*}=-\left(R+B^{T} S_{k+1} B\right)^{-1} B^{T} S_{k+1} A x_{k}^{*}=K_{k} x_{k}^{*}
$$

Close loop system transition matrix can be written using new feedback matrix in (59),

$$
A_{c}=A+B K_{k}
$$

So, the new CLF gradient $\Delta V(x)$ becomes,

$$
\begin{aligned}
\Delta V(x) & =V\left(x_{k+1}\right)-V\left(x_{k}\right) \\
& =x^{T} A_{c} S A_{c} x-x^{T} S x \\
& =x^{T}\left(A_{c} S A_{c}-S\right) x
\end{aligned}
$$

The middle term $\left(A_{c} S A_{c}-S\right)$ corresponds to the $Q$ term in the classical LQR performance index in (37). since $\Delta V(x)<0$, a positive $\mathrm{Q}$ matrix is constructed as,

$$
\begin{aligned}
Q= & S-A_{c} S A_{c} \\
= & S-A^{T} S A- \\
& -\left(R+B^{T} S_{k+1} B\right)^{-1} A^{T}\left(S_{k+1} B B^{T} S_{k+1}\right) A
\end{aligned}
$$

After we form the quantized LQR law, we define the control inputs with boundaries. Let $V(x)=x_{k}^{T} S_{k} X_{k}$ a CLF with $S_{k}>0$ and positive semi-definite Riccati matrix. A quantizer $f: X \mapsto U, f(x)=u$ is defined and the control law is,

$$
U=\left\{ \pm u_{i}: u_{i+1}=\rho u_{i}, i \in \mathbb{Z}\right\}
$$

Control law satisfies an interval, which is the solution of the $\Delta V(x)=0$. If we extend (57),

$$
\begin{aligned}
\Delta V(x)= & V\left(x_{k+1}\right)-V\left(x_{k}\right) \\
= & x_{k+1}^{T} S_{k+1} x_{k+1}-x_{k}^{T} S_{k+1} x_{k} \\
= & x^{T}\left(A^{T} S_{k} A-S_{k}\right) x+ \\
& +2 x^{T} A^{T} S_{k} B u_{k}+u_{k}^{T} B^{T} S_{k} B u_{k}
\end{aligned}
$$

$u^{(1)}$ and $u^{(2)}$ are the roots of the Eq. 66 and one can found the solution as,

$$
u^{(1),(2)}=\frac{B^{T} S_{k} A Q^{-1} A^{T} S_{k} B}{B^{T} S_{k} B} \pm \sqrt{\frac{B^{T} S_{k} A Q^{-1} A^{T} S_{k} B}{B^{T} S_{k} B}}
$$

These roots are the control boundaries for the quantized controller. Now, our optimization problem can be expressed as follows. We are trying to find the coarsest quantizer that satisfies the control boundaries $\left[u_{k}^{(1)}, u_{k}^{(2)}\right]$ and decreases the performance index in every step ( i.e. $\forall k \Delta V\left(x_{k}\right)<0$ ). We define the performance criteria as the minimum energy control that stabilizes the system.

$$
\min _{x_{k+1}=A x_{k}+B u_{k}} \text { stable } \sum_{k=0}^{\infty} u_{k}^{2}
$$

Positive semi-define Riccati equation for this system is,

$$
S_{k}^{*}=A^{T} S_{k}^{*} A-\left(B^{T} S_{k}^{*} B+1\right)^{-1} A^{T} S_{k}^{*} B B^{T} S_{k}^{*} A
$$

besides, (69) also gives the same solution of the following LQR problem,

$$
\min _{x_{k+1}=A x_{k}+B u_{k}} \text { stable } \sum_{k=0}^{\infty} u_{k}^{2}=x^{T} R x
$$

where $R=S_{k}^{*}$. State feedback gain is also evaluated as,

$$
K_{l q r}=\left(B^{T} R_{k} B\right)^{-1} B^{T} R_{k} A
$$




\section{RESUlTS AND Discussion}

First simulation results are aimed to show the superiority of the quantized LQR (qLQR) to the traditional LQR approach. In Fig. 12 generated control inputs for the same system in traditional LQR and $\mathrm{qLQR}$ are compared. While the control inputs nearly have the same values, $\mathrm{qLQR}$ control signal has an additional feature, which is the upper and lower boundary stability thresholds from (67).

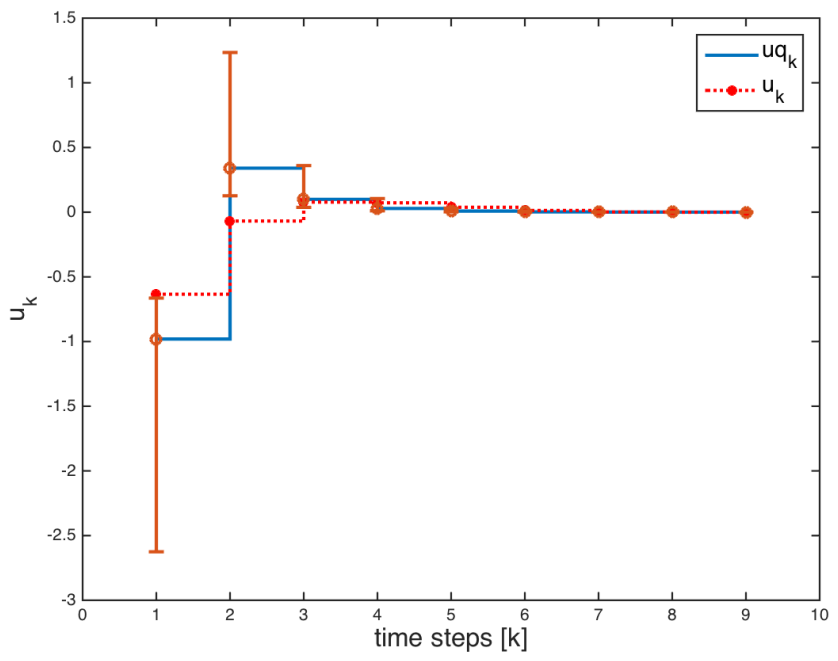

Fig. 12. Comparison between the LQR and qLQR control inputs. Control signal of the $\mathrm{qLQR}$ has additional upper and lower boundaries.

System outputs for the controller inputs in Fig. 12 is showed in Fig. 13. One can see that the system controlled with $\mathrm{qLQR}$ controller is stabilized faster than the traditional one.

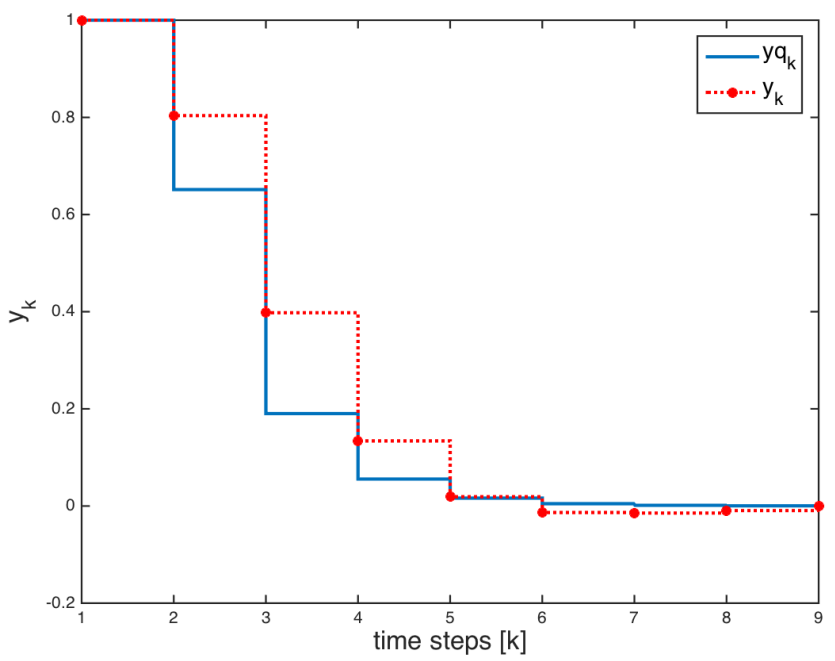

Fig. 13. Comparison between the $L Q R$ and $q L Q R$ system outputs. System controlled with $\mathrm{qLQR}$ is stabilized faster than the traditional one.

Red rectangles show the obstacle grids, and blue rectangles show the path allocations like before. In the beginning, grid graphs of both dimensions $\left(G_{x}, G_{y}\right)$ have one root note with two siblings. Thus, the ROI is separated into two halves, and very coarse representation occurs for obstacles and the path which is shown in Fig. 14(a). Using the algorithms that are given in the proposed paper, dimensions are divided into different partitions on the existence of obstacles in the path route locations. This effect can be seen clearly in Fig. 14(b). While the left half of the obstacle grids remain coarser, the right half plane grids are denser due to the path route passes nearby. This procedure proceeds continuously in real-time, so it is convenient for representing both static and dynamic objects in the framework. This approach has a disadvantage when the ROI is selected with large scale with a long path. In this case, further objects that intersect with the path may affect the spatial sampling rate along their axes. This drawback can be solved by using cascade ROI areas or setting the path spline only in local waypoints. Final results are the given for the implementation of the qLQR to the given path in the Fig. 14 . Linearized system states are specified as the $\mathrm{x}$ position, $\mathrm{y}$ position and the heading angle $x_{k}=\left[x_{i}, y_{i}, \phi_{i}\right]$. All states are gradually converged to the reference points. Gradual amplitude changes in the system states for a small route is given in Fig. 15. Besides, phase vector representation(position and the heading angle) of the states is shown in Fig. 16

\section{CONCLUSiON}

In this paper, we proposed a new framework for robot navigation problem concerning the asymptotical stability of the system with input and output quantizers. In control theory, hybrid or switched systems literature produce multiple approaches to solve stability and control under nonlinearity for many industrial usages, including robotics. However, these application areas on robotics restricted with only control designs or optimizations on multi-robot swarm formations. Using quantization for representing the occupancy grid maps and control the discontinuous dynamics of robot systems are novel to this study.

First, we have given theoretical backgrounds for quantization as a projection to robot navigation problem. Quantized control framework is represented in general and in detail as input and output quantizers. We have explained the controller used in output quantizer, denoted as $\mathrm{qLQR}$, in detail. Next, we have derived the upper and lower boundaries for the control signal that ensures asymptotical stability for the system. The results are given both in the simulation environment and realtime experiments including the comparison between traditional LQR and qLQR for an arbitrary run. In this particular application of the framework with $L Q R$ derived qLQR quantizers has limitations likely the same as the limitations in LQR controllers. Obtaining an analytical solution for the Riccati equation could be difficult in complex systems. The states that are used in the state feedback could not be observed in all situations. Thus, an observer design may have needed to be implemented to the system. However, unbounded input nature of traditional LQR methods is eliminated by using the quantizers.

In further studies, we will show the equivalence of Markov property with the state transitions between grids and redefine the structure in the probabilistic framework. Besides, we want 


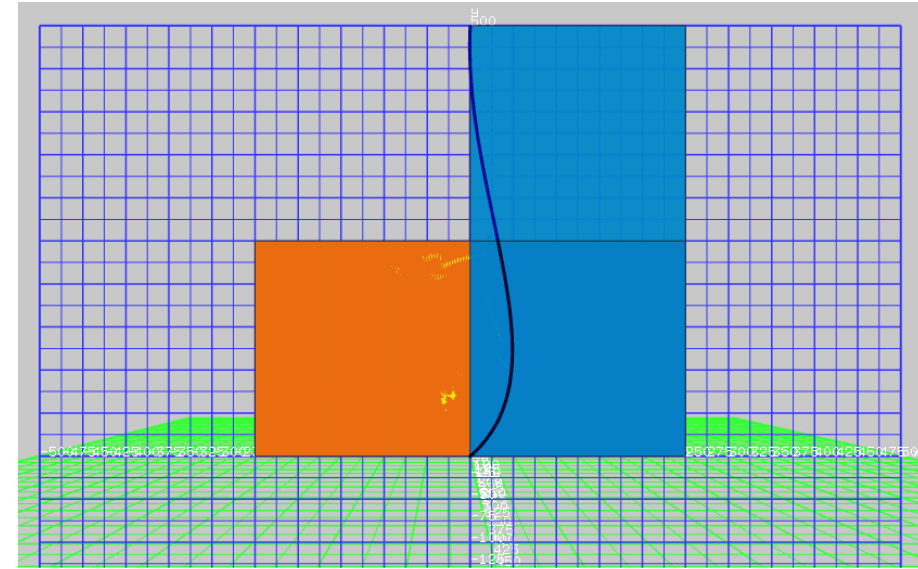

(a)

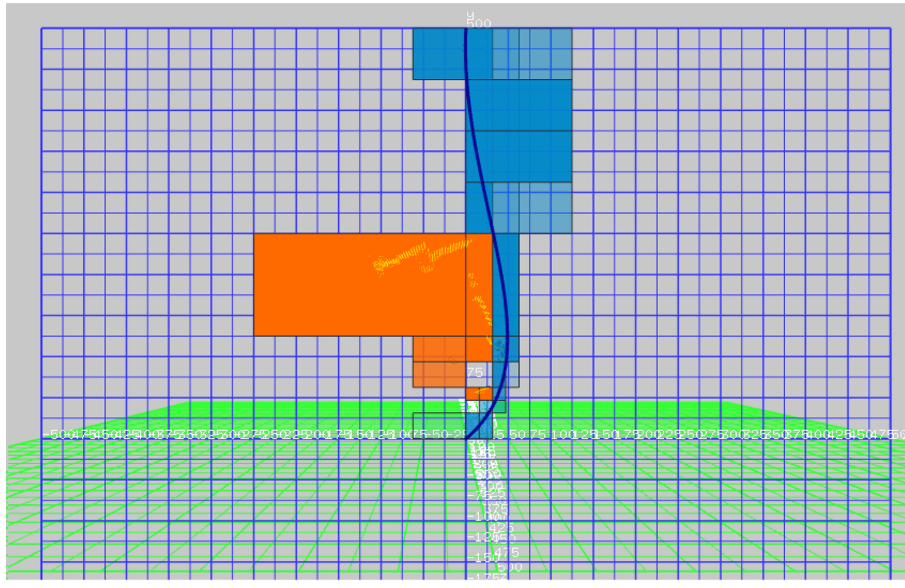

(b)

Fig. 14. Demonstration of the independent sampling rates in real life example.(a)In the beginning, both visible dimensions divide the ROI into two halves with two sibling nodes. (b) After algorithm runs, each visible dimensions are separated into different partitions due to the existence of the obstacles and intersected path splines.

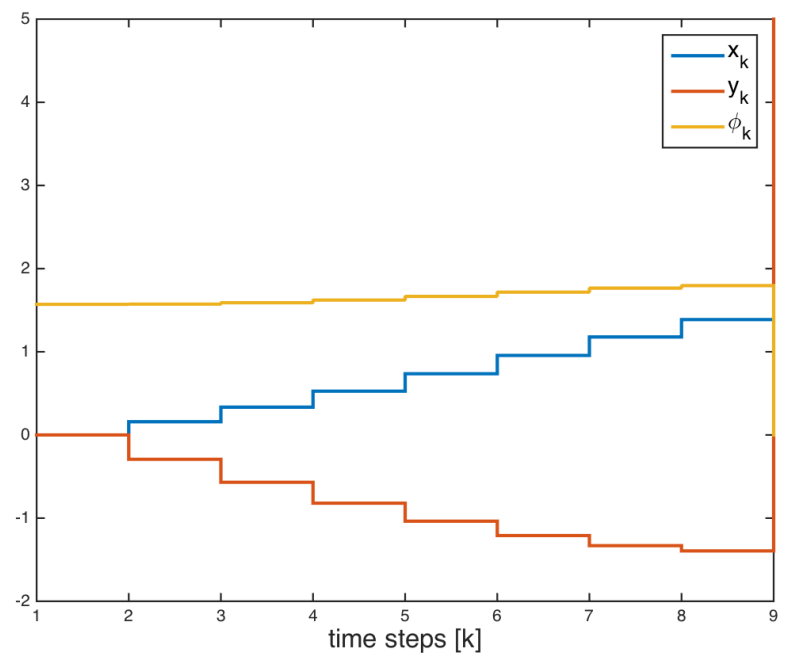

Fig. 15. System state changes with $\mathrm{qLQR}$ controller in vehicle control system.

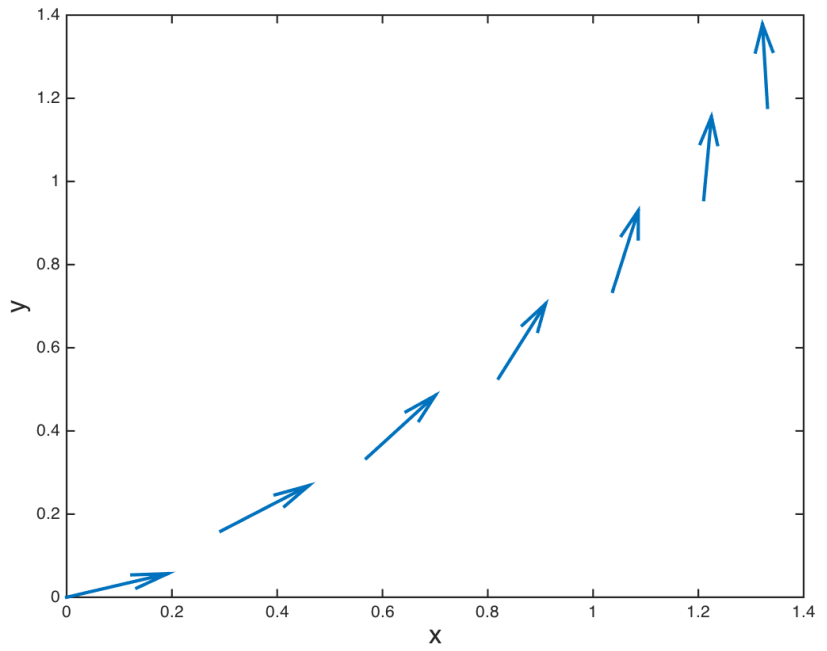

Fig. 16. Phase portrait of the qLQR controller system for $x-y$ positions of the vehicle.

to apply different state feedback methods like eigenstructure assignment to examine the feasibility of the framework.

\section{APPENDIX}

Proof for Asymptotic Stability in Quantized Feedback Control for Zoom-in/out Cases

Theorem V.1. Assume that we have the following inequality.

$$
\sqrt{\frac{\lambda_{\min }(\mathbf{P})}{\lambda_{\max }(\mathbf{P})}}>2 \Delta \frac{\|\mathbf{P B}\|\|\mathbf{K}\|}{\lambda_{\min }(\mathbf{Q})}
$$

Then there exists a quantized feedback control solution that makes the system in (26) globally asymptotically stable.

Proof: Zoom-out case: Set $u=0$, Let $\mu(0)=1$ And increase $\mu$ fast enough to dominate the rate of growth of $\left\|e^{A t}\right\|$. Then, there will be a time $t_{0} \geq 0$ such that

$$
\left\|x\left(t_{0}\right)\right\| \leq \sqrt{\frac{\lambda_{\min }(\mathbf{P})}{\lambda_{\max }(\mathbf{P})} \frac{M \mu\left(t_{0}\right)}{\|\mathbf{K}\|}}
$$

which implies that $x\left(t_{0}\right)$ belongs to ellipsoid $R_{1}\left(\mu\left(t_{0}\right)\right)$. Zoom-in case: Let's pick a $\epsilon>0$ for $t \geq t_{0}$ that holds,

$$
\sqrt{\lambda_{\min }(\mathbf{P}) M}>\sqrt{\lambda_{\max }(\mathbf{P}) Q_{u}\|K\| \Delta(1+\epsilon)}
$$

where,

$$
Q_{u}:=\frac{2\|\mathbf{P B}\|}{\lambda_{\min }(\mathbf{Q})}
$$

Let $\mu(t)=\mu(0)$ for $t \in\left[t_{0}, t_{0}+T\right)$ where $T$ is given by (35). Then, we can $x\left(t_{0}+T\right)$ belongs to the ellipsoid $R_{2}\left(\mu\left(t_{0}\right)\right)$.

For $t \in\left[t_{0}+T, t_{0}+2 T\right)$ let $\mu(t)=\Omega \mu\left(t_{0}\right)$ where,

$$
\Omega:=\frac{\sqrt{\lambda_{\max }(\mathbf{P})} Q_{u}\|K\| \Delta(1+\epsilon)}{\sqrt{\lambda_{\min }(\mathbf{P})} M}
$$


Here we have $\mu\left(t_{0}+T\right)<\mu\left(t_{0}\right)$ and $R_{2}\left(\mu\left(t_{0}\right)\right)=$ $R_{1}\left(\mu\left(t_{0}+T\right)\right)$

\section{REFERENCES}

[1] J. Keenan and J. Lewis, "Estimation with quantized measurements," in 1976 IEEE Conference on Decision and Control including the 15th Symposium on Adaptive Processes. IEEE, 1976, pp. 1284-1291.

[2] R. Curry, P. Mirchandani, and C. Price, "State Estimation with Coarsely Quantized, High-Data-Rate Measurements," IEEE Transactions on Aerospace and Electronic Systems, vol. AES-11, no. 4, pp. 613-621, 1975.

[3] C. Edwards, "Sliding mode control using only output information," in IEE Colloquium on Robust Control: Theory, Software and Applications. IEE, 1997, pp. 1010.

[4] V. I. Utkin, "Sliding Modes in Control of Electric Motors," in Sliding Modes in Control and Optimization. Berlin, Heidelberg: Springer, Berlin, Heidelberg, 1992, pp. 250-264.

[5] H. Ashrafiuon, S. Nersesov, F. Mahini, and G. Clayton, "Full state sliding mode trajectory tracking control for general planar vessel models," in 2015 American Control Conference (ACC. IEEE, 2015, pp. 5158-5163.

[6] A. Sanchez, V. Parra-Vega, C. Tang, F. Oliva-Palomo, and C. Izaguirre-Espinosa, "Continuous reactive-based position-attitude control of quadrotors," in American Control Conference - ACC 2012. IEEE, 2012, pp. 46434648.

[7] H. Lee and V. I. Utkin, "Chattering suppression methods in sliding mode control systems," Annual Reviews in Control, vol. 31, no. 2, pp. 179-188, Jan. 2007.

[8] O. Khatib, "The Potential Field Approach And Operational Space Formulation In Robot Control," in Adaptive and Learning Systems. Boston, MA: Springer, Boston, MA, 1986, pp. 367-377.

[9] M. C. Mora and J. Tornero, "Path planning and trajectory generation using multi-rate predictive Artificial Potential Fields," in IEEE/RSJ International Conference on Intelligent Robots and Systems. IEEE, 2008, pp. 2990-2995.

[10] U. Baroudi, G. Sallam, M. Al-Shaboti, and M. Younis, "GPS-free robots deployment technique for rescue operation based on landmark's criticality," in International Wireless Communications and Mobile Computing Conference (IWCMC). IEEE, 2015, pp. 367-372.

[11] W. Feiten, R. Bauer, and G. Lawitzky, "Robust obstacle avoidance in unknown and cramped environments," in IEEE International Conference on Robotics and Automation. IEEE Comput. Soc. Press, 1994, pp. 2412-2417.

[12] D. Zhou and M. Schwager, "Vector field following for quadrotors using differential flatness," in IEEE International Conference on Robotics and Automation (ICRA). IEEE, 2014, pp. 6567-6572.

[13] O. Brock and O. Khatib, "High-speed navigation using the global dynamic window approach," in International Conference on Robotics and Automation. IEEE, 1999, pp. 341-346.
[14] D. Fox, W. Burgard, and S. Thrun, "The dynamic window approach to collision avoidance," IEEE Robotics \& Automation Magazine, vol. 4, no. 1, pp. 23-33, Mar. 1997.

[15] G. A. S. Pereira, M. F. M. Campos, and V. Kumar, "Decentralized algorithms for multi-robot manipulation via caging," The International Journal of Robotics Research, vol. 23, no. 7-8, pp. 783-795, 2004.

[16] H. J. Pesch and R. Bulirsch, "The maximum principle, Bellman's equation, and Carathéodory's work," Journal of Optimization Theory and Applications, vol. 80, no. 2, pp. 199-225, Feb. 1994.

[17] M. Bernardo, C. Budd, A. R. Champneys, and P. Kowalczyk, "Piecewise-smooth dynamical systems: theory and applications," 2008.

[18] J.-M. Coron and L. Rosier, "A relation between continuous time-varying and discontinuous feedback stabilization," Journal of Mathematical Systems, Estimation, and Control, vol. 4, pp. 67-84, 011994.

[19] A. Bacciotti and F. Ceragioli, "Nonpathological Lyapunov functions and discontinuous Carathéodory systems," Automatica, vol. 42, no. 3, pp. 453-458, Mar. 2006.

[20] A. F. Filippov, "Existence and General Properties of Solutions of Discontinuous Systems," in Differential Equations with Discontinuous Righthand Sides. Dordrecht: Springer, 1988, pp. 48-122.

[21] J. M. Blatt and J. D. Gray, "An elementary derivation of Pontrayagin's maximum principle of optimal control theory," The Journal of the Australian Mathematical Society. Series B. Applied Mathematics, vol. 20, no. 02, pp. 142-156, Apr. 1977.

[22] J. Cortes, "Discontinuous dynamical systems," IEEE Control Systems Magazine, vol. 28, no. 3, pp. 36-73, May 2008.

[23] D. Liberzon, Switching in Systems and Control. Springer, Jul. 2003.

[24] D. F. Delchamps, "Stabilizing a linear system with quantized state feedback," Automatic Control, IEEE Transactions on, vol. 35, no. 8, pp. 916-924, 1990.

[25] R. Brockett and D. Liberzon, "Quantized feedback stabilization of linear systems," IEEE Transactions on Robotics, vol. 45, no. 7, pp. 1279-1289, 2000.

[26] N. Elia and S. K. Mitter, "Stabilization of linear systems with limited information," IEEE Transactions on Automatic Control, vol. 46, no. 9, pp. 1384-1400, 2001.

[27] D. Liberzon, "Hybrid feedback stabilization of systems with quantized signals," Automatica, vol. 39, no. 9, pp. 1543-1554, 2003.

[28] S.-W. Yang, C.-C. Wang, and C.-H. Chang, "RANSAC matching: Simultaneous registration and segmentation," in IEEE International Conference on Robotics and Automation (ICRA 2010). IEEE, 2010, pp. 1905-1912.

[29] R. Raguram, J.-M. Frahm, and M. Pollefeys, "A Comparative Analysis of RANSAC Techniques Leading to Adaptive Real-Time Random Sample Consensus," in 
Computer Vision - ECCV 2008. Berlin, Heidelberg: Springer, Berlin, Heidelberg, Oct. 2008, pp. 500-513.

[30] J. J. Kuffner and S. M. LaValle, "RRT-connect: An efficient approach to single-query path planning," 2000 ICRA. IEEE International Conference on Robotics and Automation, vol. 2, pp. 995-1001 vol.2, 2000.

[31] N. Mukai and N. Ishii, "R-Tree Based Path Representation for Vehicle Routing Problem," in 2009 21st IEEE International Conference on Tools with Artificial
Intelligence (ICTAI). IEEE, 2009, pp. 758-761.

[32] S. Karaman, M. R. Walter, A. Perez, E. Frazzoli, and S. Teller, "Anytime Motion Planning using the RRT*," in 2011 IEEE International Conference on Robotics and Automation (ICRA). IEEE, 2011, pp. 1478-1483.

[33] M. Otte and E. Frazzoli, "RRTX: Asymptotically optimal single-query sampling-based motion planning with quick replanning," The International Journal of Robotics Research, vol. 35, no. 7, pp. 797-822, 2015. 